\title{
Association of Genes implicated in primary angle-closure Glaucoma and the ocular biometric parameters of anterior chamber depth and axial length in a northern Chinese population
}

Shaolin Wang ${ }^{1,3+}$, Wenjuan Zhuang ${ }^{1 *+}$, Jianqing $\mathrm{Ma}^{2}$, Manyun $\mathrm{Xu}^{1}$, Shunyu Piao ${ }^{3}$, Juan $\mathrm{Hao}^{4}$, Wen Zhang ${ }^{1}$, Hao $\mathrm{Chi}^{5}$, Zhongqi Xue ${ }^{1}$ and Shaoping $\mathrm{Ha}^{1}$

\begin{abstract}
Background: The membrane frizzled-related protein (MFRP) gene is involved in axial length (AL) regulation and MFRP mutations cause nanophthalmos; also, the hepatocyte growth factor (HGF) gene is reported to result in morphologic changes of the anterior segment and abnormal aqueous regulation that increases the risk of primary angle-closure glaucoma (PACG), while the zinc ring finger 3 (ZNRF3) gene is associated with AL. The present study investigated the association of single nucleotide polymorphisms (SNPs) in ZNRF3, HGF and MFRP with PACG in a northern Chinese population, as well as the association of these SNPs with the ocular biometric parameters of anterior chamber depth (ACD) and AL.
\end{abstract}

Methods: A total of 500 PACG patients and 720 controls were recruited. All individuals were genotyped for 12 SNPs in three genes (rs7290117, rs2179129, rs4823006 and rs3178915 in ZNRF3; rs5745718, rs12536657, rs12540393, rs17427817 and rs3735520 in HGF, rs2510143, rs36015759 and rs3814762 in MFRP) using an improved multiplex ligation detection reaction (iMLDR) technique. Genotypic distribution was analyzed for Hardy-Weinberg equilibrium. Differences in the allelic and genotypic frequencies were evaluated and adjusted by age and sex. Linkage disequilibrium (LD) patterns were tested and haplotype analysis was conducted by a logistic regression model. Generalized estimation equation (GEE) analysis was conducted using SPSS for primary association testing between genotypes and ocular biometric parameters. Bonferroni corrections for multiple comparisons were performed, and the statistical power was calculated by power and sample size calculations.

Results: The rs7290117 SNP in ZNRF3 was significantly associated with the AL, with a $p$-value of 0.002 . We did not observe any significant associations between the SNPs and PACG or ACD. In a stratification analysis by ethnicity, rs12540393 and rs17427817 in HGF showed a nominal association with PACG in the Hui cohort, although significance was lost after correction.

* Correspondence: zh_wenj@163.com

'Shaolin Wang and Wenjuan Zhuang contributed equally to this work.

${ }^{1}$ People's Hospital of Ningxia Hui Autonomous Region, Ningxia Eye Hospital

(First Affiliated Hospital of Northwest University For Nationalities), 936 Huang

He East Road, Yinchuan 750011, China

Full list of author information is available at the end of the article

(c) The Author(s). 2018 Open Access This article is distributed under the terms of the Creative Commons Attribution 4.0 International License (http://creativecommons.org/licenses/by/4.0/), which permits unrestricted use, distribution, and reproduction in any medium, provided you give appropriate credit to the original author(s) and the source, provide a link to the Creative Commons license, and indicate if changes were made. The Creative Commons Public Domain Dedication waiver (http://creativecommons.org/publicdomain/zero/1.0/) applies to the data made available in this article, unless otherwise stated. 
(Continued from previous page)

Conclusions: The present study suggests rs7290117 in ZNRF3 may be involved in the regulation of $A L$, though our results do not support a contribution of the SNPs we tested in ZNRF3, HGF and MFRP to PACG in northern Chinese people. Further studies in a larger population are warranted to confirm this conclusion.

Keywords: ZNRF3, HGF, MERP, PACG, ACD, AL, Single nucleotide polymorphisms

\section{Background}

Primary angle-closure glaucoma (PACG) is a subtype of glaucoma, characterized by appositional approximation or contact between the iris and trabecular meshwork [1] and is considered to be the most common cause of bilateral glaucoma blindness worldwide [2]. Epidemiological studies have revealed that most PACG cases are in Asia [3], especially in China [4]. PACG has been recognized to be a multifactorial disease, and obvious racial differences [5] and family aggregation [6] have been confirmed in its prevalence, which suggests that genetic factors may play an important role in its pathogenesis. Up until now, 2 genome-wide association studies (GWAS) on PACG have been conducted and 8 genetic loci showed strong associations with the disease [7, 8]. In another GWAS on anterior chamber depth (ACD), the rs1401999 locus in the ABCC5 gene was also found to be associated with PACG [9]. However, these genes only partly explain the genetic predisposition to PACG.

Furthermore, the membrane frizzled-related protein (MFRP) gene was related to nanophthalmos [10] while the hepatocyte growth factor $(H G F)$ gene was reported to be associated with hyperopia [11], and both nanophthalmos and hyperopia are important risk factors for PACG [10, 12]. Meanwhile, in previous studies, the association between HGF and PACG has been evaluated in two different populations by the candidate gene approach $[13,14]$ and validated in a meta-analysis by Rong et al., although sample sizes were relatively small compared with the GWAS, which might lead to false-positive signals [15]. In addition, the zinc ring finger 3 (ZNRF3) gene was confirmed to be associated with axial length (AL) in a GWAS meta-analysis [16]. Consequently, the aim of this study was to evaluate the association of the three susceptibility genes with PACG in a northern Chinese population. In essence, we were interested in the association between these single nucleotide polymorphisms (SNPs) and the ocular biometric parameters of $\mathrm{ACD}$ and AL.

\section{Methods}

\section{Subjects}

A total of 500 cases with PACG and 720 ethnic-matched controls were recruited from the northern regions of China. The study was approved by the local hospital's ethics committee and met the tenets of the Declaration of Helsinki. Informed consent was obtained from all subjects prior to the study. Comprehensive ophthalmic examinations for each participant were performed, including best-corrected visual acuity, intraocular pressure (IOP) measurement, slit lamp biomicroscopy, fundus photography, visual field, gonioscopy and ultrasound biomicroscopy. ACD and AL were measured by IOL Master. Five readings were obtained and the mean value was used for further statistical analysis. PACG patients were diagnosed by fulfilling all of the following criteria: IOP of more than $21 \mathrm{mmHg}$; the presence of at least two quadrants of closed angle in which the trabecular meshwork was not visible on gonioscopy; the presence of glaucomatous damage to the optic nerve with a cup-to-disc ratio $\geq 0.7$ and peripheral visual loss, in accord with the International Society of Geographical and Epidemiological Ophthalmology (ISGEO) [17]. The controls were required to have none of the above characteristics and have open angles verified by gonioscopy, no known family history of glaucoma and previous glaucomatous or cataractous operations, no other ophthalmic diseases besides mild senile cataracts. Participants with secondary angle-closure glaucoma caused by trauma, uveitis, or neovascularization, were excluded.

\section{DNA extraction}

Peripheral venous blood samples were collected from all participants and genomic DNA was isolated from the blood samples utilizing the Simgen DNA Blood Mini Kit (Simgen, Hangzhou, China) in accordance with the manufacturer's protocol. The extracted DNA was eluted in TE buffer (10 mM Tris-HCl, 0.5 mM EDTA, pH 9.0) and then stored at $-80^{\circ}$ until use after the A260/A280 optical density was measured with Nanodrop2000 (Thermo Fisher Scientific Inc., Wilmington, DE, USA).

\section{SNP selection and genotyping}

Since associations or possible associations between our target genes and PACG were reported in previous studies $[13,14,18-20]$, a total of 12 SNPs were chosen as candidates. They were rs7290117, rs2179129, rs4823006 and rs3178915 in ZNRF3; rs5745718, rs12536657, rs12540393, rs17427817 and rs3735520 in HGF; rs2510143, rs36015759 and rs3814762 in MFRP. All SNPs were genotyped by Genesky Biotechnologies 
Inc. (Shanghai, China) using an improved multiplex ligation detection reaction (iMLDR) technique.

\section{Statistical analysis}

Demographic differences between the cases and controls were performed using the SPSS software (version 17.5: SPSS Science, Chicago, IL), the differences in sex and ethnicity were assessed by the $x^{2}$ test and the differences in age were assessed by $\mathrm{T}$ test. Each SNP was appraised for compliance with Hardy-Weinberg equilibrium (HWE) using the $\mathrm{X}^{2}$ test. The genetic association analyses as well as the meta-analysis were conducted using PLINK (version 1.07; http://zzz.bwh.harvard.edu/plink/ index.shtml, in the public domain). Allelic and genotypic frequency differences of a given SNP between the PACG patients and the controls were evaluated and adjusted by age and sex using a logistic regression model. Meanwhile, the adjusted odds ratios (ORs) and the corresponding 95\% confidence intervals (CIs) for associations were also presented. Linkage disequilibrium (LD) patterns were tested with Haploview 4.2 software (Daly Lab at the Broad Institute, Cambridge, MA), and haplotype analysis was also conducted by a logistic regression model and adjusted for age and sex. Generalized estimation equation (GEE) analysis with an unstructured working correlation matrix modeling for a trend-per-copy effect on the minor allele (coding 0 for the wild-type genotype, 1 for heterozygous genotype, and 2 for homozygous genotype for the minor allele) was performed using SPSS for association testing between genotypes and ocular biometric parameters. In this analysis, the genotypes were treated as covariates, $\mathrm{ACD}$ and $\mathrm{AL}$ were control variables of each other, and age and sex were adjusted. Bonferroni correction was performed if a positive association (a $p$-value of less than $0.05)$ was found in the initial analysis. The statistical power was calculated by the Power and Sample Size Calculation (PS; version 3.1.232).

\section{Results}

This study comprised 500 PACG patients (147 males and 353 females; 93 Hui and 407 Han) and 720 control subjects (332 males and 388 females; 129 Hui and 591 Han) from the northern regions of China. There were no significant differences in ethnicity between cases and controls. However, the control subjects were significantly older (mean age $71.82 \pm 7.2$ years vs. $63.77 \pm 9.576$ years; $p=0.000$, which was an intentional design for this age-related disease) and included less women (53.9\% vs. $70.6 \% ; \mathrm{p}=0.000$ ) than the case group (Table 1).

The genotyping call rates for the 12 SNPs in both case and control groups were more than $99 \%$ and their allele distributions were within HWE $(P>0.05)$ (Table 2). The distributions of the allele and genotype frequencies of all SNPs were not significantly different between PACG
Table 1 Demographic characteristics of PACG cases and controls

\begin{tabular}{llll}
\hline & Cases & Controls & $P$ \\
\hline Number & 500 & 720 & \\
Age, y (Mean \pm SD) & $63.77 \pm 9.576$ & $71.82 \pm 7.2$ & $0.000 \#$ \\
$\begin{array}{l}\text { Sex, } n \text { (\%) } \\
\quad \text { Male }\end{array}$ & $147(29.4)$ & $332(46.1)$ & $0.000^{*}$ \\
$\quad$ Female & $353(70.6)$ & $388(53.9)$ & \\
Ethnicity, $n$ (\%) & & & $0.761^{*}$ \\
$\quad$ Han & & & \\
Hui & $407(81.4)$ & $129(17.9)$ & \\
\hline
\end{tabular}

\#The $p$-value was tested by T-test

*The $p$-value was assessed by $\times 2$ test

patients and control subjects. Haplotype analysis was also performed and none of the common haplotypes showed any significant differences between PACG patients and control subjects (Fig. 1, Table 3). Meanwhile subanalysis was also performed within the Hui PACG cases versus Hui controls and Han PACG cases versus Han controls since the participants recruited included two peoples. Rs12540393 and rs17427817 in HGF, with the same $p$-value of 0.019 , were associated with PACG in the Hui cohort after correction for age and sex using logistic regression and the frequencies of the minor $\mathrm{C}$ allele of rs12540393 as well as rs17427817 were less in the PACG group than in the control group. However, the significance was lost after Bonferroni correction. None of the remaining SNPs and haplotypes were associated with PACG in either the Hui or Han cohort. We amalgamated the results of the separate analyses of the two different ethnicities, and the meta-analysis $p$-values were almost the same as the initial overall analysis (Table 4).

Furthermore, in association testing between the 12 SNP genotypes and AL and ACD ocular biometric parameters using GEE tests, we found rs7290117 in ZNRF3 was associated significantly with the AL with a $p$-value of 0.002 (adjusted $p$-value was 0.024 ), the variant allele of which may have the effect of making the AL shorter $(\beta=-0.169)$ (Table 5).

The power varies between the 12 SNPs due to the difference of their minor allele frequency (MAF). Therefore, assuming an allelic OR of 1.5, our sample size provides more than $95 \%$ statistical power to detect a significant association at an $\alpha$ level of 0.05 with the exception of the SNP rs7290117, which has 77\% statistical power to detect a significant association in the same conditions.

\section{Discussion}

PACG is a multifactorial disease, and both genetic and environmental factors are significant to its progression [1]. Candidate gene approaches have been used to 
Table 2 Association results of target SNPs with PACG after adjustment for age and sex

\begin{tabular}{|c|c|c|c|c|c|c|c|c|c|c|c|c|}
\hline \multirow[t]{2}{*}{ GENE } & \multirow[t]{2}{*}{ SNP } & \multirow[t]{2}{*}{$\mathrm{CHR}$} & \multirow[t]{2}{*}{$\mathrm{BP}$} & \multirow[t]{2}{*}{ Minor allele } & \multicolumn{2}{|c|}{ 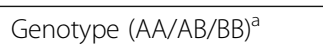 } & \multicolumn{2}{|l|}{ MAF } & \multicolumn{2}{|l|}{ HWE- $p$} & \multirow[t]{2}{*}{ OR $(95 \% \mathrm{Cl})$} & \multirow[t]{2}{*}{$P$} \\
\hline & & & & & Case & Control & Case & Control & Case & Control & & \\
\hline ZNRF3 & rs7290117 & 22 & $29,450,856$ & T & $427 / 72 / 1$ & $632 / 84 / 4$ & 0.074 & 0.064 & 0.5056 & 0.524 & 1.306(0.9007 1.894) & 0.159 \\
\hline ZNRF3 & rs2179129 & 22 & $29,450,923$ & G & $179 / 238 / 83$ & 236/347/137 & 0.404 & 0.431 & 0.7813 & 0.6487 & $0.9095(0.7571 \sim 1.092)$ & 0.3105 \\
\hline ZNRF3 & rs4823006 & 22 & $29,451,671$ & G & $130 / 252 / 118$ & 200/381/139 & 0.488 & 0.458 & 0.9287 & 0.08442 & $1.166(0.9671 \sim 1.407)$ & 0.1073 \\
\hline ZNRF3 & rs3178915 & 22 & $29,453,027$ & A & $168 / 243 / 88$ & 252/358/108 & 0.419 & 0.400 & 1 & 0.3128 & 1.085 (0.8992 1.309) & 0.3954 \\
\hline HGF & rs5745718 & 7 & $81,347,548$ & T & $366 / 124 / 10$ & $537 / 172 / 11$ & 0.144 & 0.134 & 1 & 0.6314 & $0.9475(0.7256 \sim 1.237)$ & 0.6924 \\
\hline HGF & rs12536657 & 7 & $81,350,208$ & A & $363 / 126 / 10$ & $534 / 174 / 11$ & 0.146 & 0.136 & 1 & 0.5287 & $0.9514(0.7292 \sim 1.241)$ & 0.7132 \\
\hline HGF & rs12540393 & 7 & $81,364,187$ & C & $342 / 143 / 14$ & $513 / 191 / 16$ & 0.171 & 0.155 & 1 & 0.8865 & 0.9266 (0.7216 1.19) & 0.5503 \\
\hline HGF & rs 17427817 & 7 & $81,364,435$ & C & $342 / 144 / 14$ & $512 / 192 / 16$ & 0.172 & 0.155 & 0.8764 & 0.7774 & 0.9249 (0.7203 1.188) & 0.5405 \\
\hline HGF & rs3735520 & 7 & $81,400,939$ & A & 139/258/103 & 227/349/144 & 0.464 & 0.442 & 0.4199 & 0.6505 & 1.191 (0.9897 1.433) & 0.0643 \\
\hline MFRP & rs2510143 & 11 & $119,216,231$ & A & $372 / 119 / 9$ & $515 / 186 / 19$ & 0.137 & 0.156 & 1 & 0.6699 & 0.8559 (0.6603 1.109) & 0.2396 \\
\hline MFRP & rs36015759 & 11 & $119,216,279$ & A & 289/178/33 & $425 / 252 / 43$ & 0.244 & 0.235 & 0.4665 & 0.4694 & 1.085 (0.8794 1.338) & 0.4478 \\
\hline MFRP & rs3814762 & 11 & $119,216,504$ & T & $328 / 151 / 21$ & $479 / 211 / 29$ & 0.193 & 0.187 & 0.4746 & 0.3285 & 0.946 (0.7259 1.189) & 0.6337 \\
\hline
\end{tabular}

${ }^{\mathrm{a} A}$ represents the wild-type allele, B represents the minor allele; CHR chromosome, BP base pair position, MAF minor allele frequency, HWE- $p$ the $p$-value of Hardy-Weinburg equilibrium, $O R$ odds ratio, $C l$ confidence interval

$P$-value, $\mathrm{OR}$, and $\mathrm{Cl}$ were calculated with a logistic regression model by adjusting for age and sex

explore the genetic architecture of glaucoma and some possible susceptibility genes have been reported. In the present study, we chose three genes that were previously reported as having an association with regulation of AL $[10,16]$ or hyperopia [11] to evaluate the association between these genes and PACG in a northern Chinese cohort. Consequently, we did not observe any association between the three target genes and PACG. However, rs7290117 in ZNRF3 was validated to be significantly associated with the AL by the GEE method [21, 22], which is suitable for statistical analysis of correlated data since binocular biometric parameters can better reflect the genetic characteristics. To the best of our knowledge, this is the first study to investigate the association of the AL-related gene ZNRF3 with PACG.

PACG patients have similar anatomical features, such as shallow anterior chambers and short AL [2]. Recently, Cheng et al. found rs12321 in ZNRF3 was associated with AL in a GWAS meta-analysis [16], and proteins encoded by ZNRF3 are directly involved in the Wnt signaling pathway [23], which is a significant pathway in vertebrate eye development [24]. Shi et al. evaluated the association between ZNRF3 and primary angle-closure $(\mathrm{PAC})$ in a Chinese cohort and found no association between them [20]. In our study, we also failed to find any association between ZNRF3 and PACG. Nevertheless,

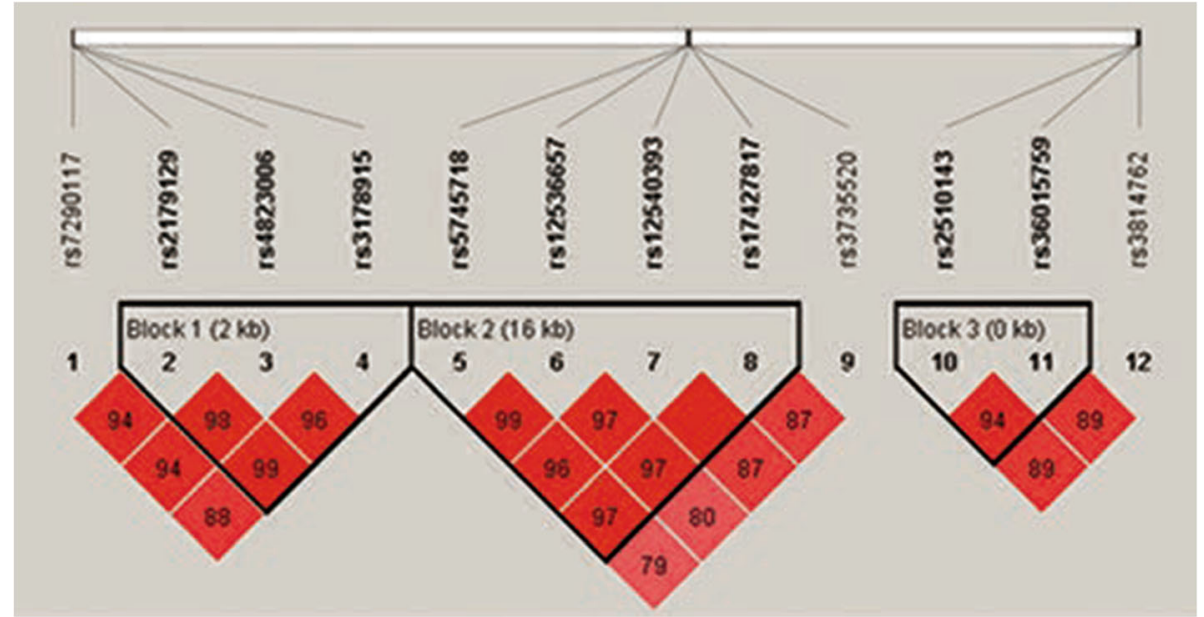

Fig. 1 Three Haplotype Blocks of the 12 Target SNPs. Nine target SNPs are presented to the three haplotype blocks in HapMap CHB cohort combined of PACG and control, which were determined by the Haploview 4.2 program. Darker shades suggested higher linkage disequilibrium 
Table 3 Haplotype analysis of the target genes in PACG and control cohorts

\begin{tabular}{|c|c|c|c|c|c|c|}
\hline Block & SNPS & Haplotype & Freq of cases (\%) & Freq of controls (\%) & OR & $P$-value \\
\hline \multirow[t]{4}{*}{ Block 1} & \multirow[t]{4}{*}{ rs2179129, rs4823006, rs3178915 } & AGA & 41.77 & 38.74 & 1.15 & 0.153 \\
\hline & & AGG & 6.92 & 6.54 & 1.15 & 0.476 \\
\hline & & GAG & 40.29 & 42.48 & 0.924 & 0.403 \\
\hline & & AAG & 10.69 & 10.48 & 0.96 & 0.785 \\
\hline \multirow[t]{3}{*}{ Block 2} & \multirow[t]{3}{*}{ rs5745718, rs12536657, rs12540393, rs 17427817} & TACC & 13.81 & 13.09 & 0.926 & 0.575 \\
\hline & & GGCC & 3.02 & 2.02 & 0.961 & 0.895 \\
\hline & & GGTG & 82.25 & 84.26 & 1.05 & 0.687 \\
\hline \multirow[t]{3}{*}{ Block 3} & \multirow[t]{3}{*}{ rs2510143, rs36015759 } & GA & 24.18 & 23.35 & 1.1 & 0.396 \\
\hline & & $A G$ & 13.48 & 15.41 & 0.857 & 0.251 \\
\hline & & GG & 62.12 & 61.05 & 1.01 & 0.907 \\
\hline
\end{tabular}

OR and $P$-value were calculated with the logistic regression model by adjusting for age and sex

we found rs7290117 in ZNRF3 was significantly associated with the $\mathrm{AL}$, which is in line with a previous GWAS meta-analysis [16].

The HGF gene has been confirmed to be involved in the emmetropization process of the eye and stimulating the growth and migration of many eye tissues [25-27]. A recent study found some SNPs of the HGF gene were associated with susceptibility to hyperopia [11]. Several SNPs of the HGF gene were also associated with PACG in different populations [13, 14], Awadalla et al. found four SNPs (rs5745718, rs12536657, rs12540393 and rs17427817) in $H G F$ were significantly associated with PACG in a case-control study comprised of 106 patients and 204 controls in the Nepalese population [13], Jiang et al. identified two SNPs (rs5745718 and rs1742817) and a haplotype in HGF associated with PACG in a case-control study comprised of 238 patients and 287 controls from the east of China [14], and Rong et al. confirmed the association between the SNPs rs5745718 as well as rs1742817 and PACG through a meta-analysis [15]. In our study, we found rs12540393 and rs17427817 in $H G F$ showed a nominal association with PACG in the Hui cohort, and the odds ratios of the two SNPs were contrary to previous findings and the Han cohort. Although the significance was lost after Bonferroni correction, to some extent, such results reflected ethnic differences in disease pathogenesis and implied the association of markers was diverse in different ethnic groups. Considering that the small sample size of the Hui cohort in our study is likely to result in false-positive consequences, the relationship between HGF and PACG in different populations still needs further study.

$M F R P$ is located on human chromosome 11q23.3, and the $\mathrm{COOH}$ terminal domain of MFRP is known to be

Table 4 Associations for target SNPs between cases and controls in different ethnicities as well as the meta-analysis results

\begin{tabular}{|c|c|c|c|c|c|c|c|c|c|c|c|}
\hline \multirow[t]{2}{*}{ SNP } & \multicolumn{2}{|c|}{ MAF-case } & \multicolumn{2}{|c|}{ MAF-control } & \multicolumn{2}{|l|}{ OR $(95 \% \mathrm{Cl})$} & \multicolumn{2}{|l|}{$p$} & \multirow{2}{*}{$\begin{array}{l}P_{-} \\
\text {meta }^{a}\end{array}$} & \multirow[t]{2}{*}{$1^{2}$} & \multirow[t]{2}{*}{ P-het } \\
\hline & $\mathrm{HUI}$ & HAN & $\mathrm{HUI}$ & HAN & $\mathrm{HUI}$ & HAN & $\mathrm{HUI}$ & HAN & & & \\
\hline rs7290117 & 0.06452 & 0.07617 & 0.05118 & 0.06684 & $2.103(0.8305 \sim 5.237)$ & $1.151(0.7692 \sim 1.723)$ & 0.1169 & 0.4937 & 0.184 & 21.57 & 0.2588 \\
\hline rs2179129 & 0.4409 & 0.3956 & 0.4488 & 0.4272 & $0.877(0.5951 \sim 1.293)$ & $0.9251(0.7511 \sim 1.139)$ & 0.5073 & 0.4638 & 0.3643 & 0 & 0.7915 \\
\hline rs4823006 & 0.457 & 0.4951 & 0.4882 & 0.4509 & 0.9995 (0.667 1.498) & $1.194(0.9665 \sim 1.475)$ & 0.998 & 0.1002 & 0.1359 & 0 & 0.4326 \\
\hline rs3178915 & 0.3925 & 0.4261 & 0.4252 & 0.3947 & $0.9102(0.5945 \sim 1.394)$ & $1.123(0.9105 \sim 1.385)$ & 0.6652 & 0.2783 & 0.4353 & 0 & 0.3858 \\
\hline rs5745718 & 0.1022 & 0.1536 & 0.1732 & 0.1261 & $0.573(0.3114 \sim 1.054)$ & $1.061(0.784 \sim 1.435)$ & 0.07339 & 0.7022 & 0.5334 & 68.41 & 0.0752 \\
\hline rs12536657 & 0.1022 & 0.1564 & 0.1732 & 0.128 & $0.5612(0.3048 \sim 1.033)$ & $1.074(0.7944 \sim 1.452)$ & 0.06371 & 0.6423 & 0.5369 & 71.26 & 0.0621 \\
\hline rs12540393 & 0.1183 & 0.1835 & 0.2087 & 0.143 & $0.5067(0.2872 \sim 0.8939)$ & 1.077 (0.8089 1.433) & 0.01892 & 0.6131 & 0.4807 & 81.32 & 0.0207 \\
\hline rs17427817 & 0.1183 & 0.1843 & 0.2087 & 0.1438 & $0.5067(0.2872 \sim 0.8939)$ & $1.069(0.8035 \sim 1.421)$ & 0.01892 & 0.6485 & 0.4782 & 81.23 & 0.021 \\
\hline rs3735520 & 0.4839 & 0.4595 & 0.4094 & 0.4492 & $1.375(0.9151 \sim 2.065)$ & $1.158(0.9402 \sim 1.426)$ & 0.1254 & 0.1675 & 0.05236 & 0 & 0.4662 \\
\hline rs2510143 & 0.172 & 0.129 & 0.2047 & 0.1455 & $0.7626(0.4533 \sim 1.283)$ & $0.872(0.6455 \sim 1.178)$ & 0.3072 & 0.3722 & 0.2059 & 0 & 0.6545 \\
\hline rs36015759 & 0.2581 & 0.2408 & 0.1969 & 0.2437 & $1.526(0.938 \sim 2.481)$ & $1.005(0.7954 \sim 1.27)$ & 0.08872 & 0.9671 & 0.4457 & 57.53 & 0.1249 \\
\hline rs3814762 & 0.1452 & 0.2039 & 0.1693 & 0.1912 & $0.7363(0.412 \sim 1.316)$ & $0.988(0.7692 \sim 1.269)$ & 0.3014 & 0.9249 & 0.6413 & 0 & 0.3552 \\
\hline
\end{tabular}

MAF minor allele frequency, $O R$ odds ratio, $C l$ confidence interval, $I^{2}$ measures heterogeneity, $p$-het $p$-value for heterogeneit;

$P$-value, $\mathrm{OR}$, and $\mathrm{Cl}$ were calculated with a logistic regression model by adjusting for age and sex

${ }^{\text {a }}$-meta, $P$-value obtained by meta-analysis, if the $\mathrm{I}^{2}$ value was $\geq 50 \%$, we took the value of random-effects; otherwise, a fixed-effects model was adopted 
Table 5 Association results between the target Loci, $A L$, and $A C D$

\begin{tabular}{|c|c|c|c|c|c|c|c|c|}
\hline \multirow[t]{2}{*}{ GENE } & \multirow[t]{2}{*}{ SNP } & \multirow[t]{2}{*}{ Minor allele } & \multicolumn{3}{|c|}{$\operatorname{AL}(22.92 \pm 0.891 ; 20.01 \sim 25.51)^{a}$} & \multicolumn{3}{|c|}{$\operatorname{ACD}(2.74 \pm 0.474 ; 0.25 \sim 4.51)^{a}$} \\
\hline & & & $\bar{\beta}$ & SE & $P$ & $\bar{\beta}$ & SE & $P$ \\
\hline ZNRF3 & rs7290117 & T & -0.169 & 0.055 & 0.002 & -0.008 & 0.0312 & 0.808 \\
\hline ZNRF3 & rs2179129 & G & 0.003 & 0.0313 & 0.925 & 0.001 & 0.0157 & 0.925 \\
\hline ZNRF3 & rs4823006 & G & -0.023 & 0.0317 & 0.461 & 0.007 & 0.0159 & 0.666 \\
\hline ZNRF3 & rs3178915 & A & 0.025 & 0.0313 & 0.42 & 0.005 & 0.0161 & 0.74 \\
\hline HGF & rs5745718 & T & -0.005 & 0.0417 & 0.912 & 0.011 & 0.0243 & 0.64 \\
\hline HGF & rs12536657 & A & 0.001 & 0.0415 & 0.978 & 0.01 & 0.0243 & 0.69 \\
\hline HGF & rs12540393 & C & -0.027 & 0.0394 & 0.495 & 0.012 & 0.023 & 0.594 \\
\hline HGF & rs17427817 & C & -0.026 & 0.0393 & 0.516 & 0.011 & 0.023 & 0.647 \\
\hline HGF & rs3735520 & $A$ & -0.018 & 0.0309 & 0.568 & -0.024 & 0.0157 & 0.127 \\
\hline MFRP & rs2510143 & $A$ & -0.016 & 0.0432 & 0.714 & 0.012 & 0.0218 & 0.581 \\
\hline MFRP & rs36015759 & A & 0.04 & 0.0339 & 0.234 & 0.009 & 0.0195 & 0.654 \\
\hline MFRP & rs3814762 & T & -0.014 & 0.0362 & 0.703 & 0.002 & 0.0202 & 0.918 \\
\hline
\end{tabular}

${ }^{a}$ Numbers in parentheses indicate the Mean $\pm S D$ and the range of measured values for $A L$ or $A C D \beta$, per-allele effect in $A C D / A L, S E$ standard error for ascertainment of $\beta, P, P$-value for association adjusting for age and sex

related to the Wnt binding cysteine-rich domain of the frizzled family of transmembrane proteins which are receptors for the Wnt signaling pathway [28], a significant pathway in vertebrate eye development [24]. Mutations in MFRP were reported to cause autosomal recessive nanophthalmos, which is characterized by short AL, a small corneal diameter, a high lens/eye volume ratio, and a high degree of hyperopia [10]. Therefore, MFRP was considered to be a candidate gene for PACG as well as PAC, however, previous studies did not indicate any significant association between MFRP and PACG or PAC in different populations $[18,19,29]$, similar to our finding.

Moreover, in the present study, we failed to validate any association between the two nanophthalmos or hyperopia-related genes (MFRP, HGF) with $\mathrm{AL}$ and $\mathrm{ACD}$ or between the three target genes and PACG, since nanophthalmos shows the same characteristics as PACG of a short AL and hyperopia is an important phenotype associated with PACG. Our results suggest that the genes associated with a phenotype of a certain disease are not necessarily related to the disease itself since a disease may have many complex phenotypes, with one or some that are not equal to the disease. Exploration of a certain phenotype is only a tiny point in understanding of the disease, but the understanding of many such "tiny points" will eventually produce an objective and comprehensive understanding of the disease, as in existing genetic association studies, where the function of a single susceptibility locus may be tiny and confusing. Therefore, deeper and more extensive research is necessary. Furthermore, this study involved two ethnic groups, which might make the result of the overall analysis lack credibility. However, we performed a meta-analysis of the two different ethnic groups, and the meta-analysis $p$-values were almost the same as the initial overall analysis. This proves that our initial overall analysis results are reliable and an ethnic-matched case-control study design is feasible when it involves two different ethnicities with small sample size.

The limitation of our research is that the SNPs were chosen on the basis of previous studies but did not utilize the tagger program, which is a common method for candidate gene research and often presents different results in different populations. The SNPs selected thus may not completely represent the genes in our cohort. Therefore, tagger SNPs based on our cohort should be selected for more in-depth study based on pathogenesis in the future.

\section{Conclusions}

We conducted a case-control study of 12 SNPs among 500 PACG subjects and 720 ethnic-matched controls using a candidate gene approach. Our results do not support contribution of the SNPs we tested in ZNRF3, $H G F$ and MFRP to PACG in northern Chinese people. However we confirmed the association of rs7290117 in ZNRF3 with AL which suggests rs7290117 might be involved in the regulation of ocular biometric parameters of AL in PACG. Further studies in a larger population are needed to verify this conclusion.

\section{Abbreviations}

ACD: anterior chamber depth; AL: axial length; Cls: confidence intervals: GEE: Generalized estimation equation; GWAS: genome-wide association studies; HGF: hepatocyte growth factor; HWE: Hardy-Weinberg equilibrium; iMLDR: improved multiplex ligation detection reaction; IOP: intraocular pressure; ISGEO: International Society of Geographical and Epidemiological Ophthalmology; LD: Linkage disequilibrium; MAF: minor allele frequency; MFRP: membrane frizzled-related protein; OR: odds ratio; PACG: primary angle-closure glaucoma; SNPs: single nucleotide polymorphisms; ZNRF3: zinc ring finger 3 


\section{Acknowledgments}

The authors thank all the patients and participants.

\section{Funding}

This work was supported by grants from the National Basic Research Program of China [Program; \#81460093] and Ningxia Nature Science Funding (Grant-NZ16194) from the Department of Science and technology of Ningxia Hui Autonomous Region.

\section{Availability of data and materials}

The datasets used and/or analyzed during the current study are available from the corresponding author on reasonable request.

\section{Authors' contributions}

SW performed clinical examinations, carried out the technical work in the laboratory, wrote the manuscripts and analyzed of the data; WZ supervised the overall study, wrote the manuscripts and analyzed of the data; JM, MX, $S P, J H, W Z, H C$ and ZX carried out the technical work in the laboratory, including patient's requirements, and reviewed the files for clinical information; SH performed clinical examinations and supervised the overall study. All authors read and approved the final manuscript.

\section{Ethical approval and consent to participate}

This study was approved by the ethics committee of the People's Hospital of Ningxia Hui Autonomous Region and met the tenets of the Declaration of Helsinki, and informed consent was obtained from all of the subjects prior to the study.

\section{Consent for publication}

Not applicable.

\section{Competing interests}

The authors declare that they have no competing interests.

\section{Publisher's Note}

Springer Nature remains neutral with regard to jurisdictional claims in published maps and institutional affiliations.

\section{Author details}

${ }^{1}$ People's Hospital of Ningxia Hui Autonomous Region, Ningxia Eye Hospital (First Affiliated Hospital of Northwest University For Nationalities), 936 Huang He East Road, Yinchuan 750011, China. ${ }^{2}$ Department of Ophthalmology, Wuzhong People's Hospital, 430 Wen Wei Road, Wuzhong 751100, China. ${ }^{3}$ Clinical Medical College of Ningxia Medical University, 1160 Shengli Street, Yinchuan 750001, China. ${ }^{4}$ Department of Ophthalmology, Taiyuan Central Hospital, 1 Dong San Dao Lane, Jie Fang North Road, Taiyuan 030000, China. ${ }^{5}$ Shandong Academy of Medical Sciences, Jinan University, 18877 Jing Shi Road, Li Xia District, Jinan 250000, China.

Received: 16 July 2018 Accepted: 5 October 2018

Published online: 22 October 2018

\section{References}

1. Wright C, Tawfik MA, Waisbourd M, Katz L. Primary angle-closure glaucoma: an update. Acta Ophthalmol. 2016;94(3):217-25.

2. Sihota R, Ghate D, Mohan S, Gupta V, Pandey RM, Dada T. Ocular parameters in the subgroups of angle closure glaucoma. Eye (Lond). 2008;22(4):521-7.

3. Congdon N, Wang F, Tielsch JM. Issues in the epidemiology and population-based screening of primary angle-closure glaucoma. Surv Ophthalmol. 1992;36(6):411-23.

4. Foster PJ, Baasanhu J, Alsbirk PH, Munkhbayar D, Uranchimeg D, Johnson GJ. Glaucoma in Mongolia. A population-based survey in Hövsgöl province, northern Mongolia. Arch Ophthalmol. 1996;114(10):1235-41.

5. Wang $N$, Wu H, Fan Z. Primary angle closure glaucoma in Chinese and Western populations. Chin Med J. 2002:115:1706-15.

6. Amerasinghe N, Zhang J, Thalamuthu A, He M, Vithana EN, Viswanathan A, et al. The heritability and sibling risk of angle closure in Asians. Ophthalmology. 2011;118(3):480-5.
7. Vithana EN, Khor CC, Qiao C, Nongpiur ME, George R, Chen LJ, et al. Genome-wide association analyses identify three new susceptibility loci for primary angle closure glaucoma. Nat Genet. 2012;44(10):1142-6.

8. Khor CC, Do T, Jia H, Nakano M, George R, Abu-Amero K, et al. Genomewide association study identifies five new susceptibility loci for primary angle closure glaucoma. Nat Genet. 2016:48(5):556-62.

9. Nongpiur ME, Khor CC, Jia H, Cornes BK, Chen L, Qiao C, et al. ABCC5, a gene that influences the anterior chamber depth, is associated with primary angle closure glaucoma. PLoS Genet. 2014;10(3):e1004089.

10. Sundin $\mathrm{OH}$, Leppert GS, Silva ED, Yang JM, Dharmaraj S, Maumenee $\mid \mathrm{H}$, et al Extreme hyperopia is the result of null mutations in MFRP, which encodes a frizzled-related protein. Proc Natl Acad Sci U S A. 2005;102(27):9553-8.

11. Veerappan S, Pertile KK, Islam AF, Schäche M, Chen CY, Mitchell P, et al. Role of the hepatocyte growth factor gene in refractive error. Ophthalmology. 2010;117(2):239-45.

12. Shen L, Melles RB, Metlapally R, Barcellos L, Schaefer C, Risch N, et al. The Association of Refractive Error with Glaucoma in a multiethnic population Ophthalmology. 2016;123(1):92-101.

13. Awadalla MS, Thapa SS, Burdon KP, Hewitt AW, Craig JE. The association of hepatocyte growth factor (HGF) gene with primary angle closure glaucoma in the Nepalese population. Mol Vis. 2011;17:2248-54.

14. Jiang Z, Liang K, Ding B, Tan W, Wang J, Lu Y, et al. Hepatocyte growth factor genetic variations and primary angle-closure Glaucoma in the Han Chinese population. PLoS One. 2013:8(4):e60950.

15. Rong SS, Tang FY, Chu WK, Ma L, Yam JC, Tang SM, et al. Genetic associations of primary angle-closure disease: a systematic review and meta-analysis. Ophthalmology. 2016;123(6):1211-21.

16. Cheng CY, Schache M, Ikram MK, Young TL, Guggenheim JA, Vitart V, et al. Nine loci for ocular axial length identified through genome-wide association studies, including shared loci with refractive error. Am J Hum Genet. 2013:93(2):264-77.

17. Foster PJ, Buhrmann R, Quigley HA, Johnson GJ. The definition and classification of glaucoma in prevalence surveys. $\mathrm{Br} J$ Ophthalmol. 2002;86:238-42.

18. Awadalla MS, Burdon KP. Thapa SS, Hewitt AW. Craig JE. A cross-ethnicity investigation of genes previously implicated in primary angle closure glaucoma. Mol Vis. 2012:18:2247-54.

19. Shi H, Zhu R, Hu N, Shi J, Zhang J, Jiang $L$, et al. Association of frizzledrelated protein (MFRP) and heat shock protein 70 (HSP70) single nucleotide polymorphisms with primary angle closure in a Han Chinese population: Jiangsu eye study. Mol Vis. 2013;19:128-34.

20. Shi H, Zhang J, Zhu R, Hu N, Lu H, Yang M, et al. Primary angle closure and sequence variants within MicroRNA binding sites of genes involved in eye development. PLoS One. 2016;11(11):e0166055.

21. Zeger SL, Liang K-Y, Albert PS. Models for longitudinal data: a generalized estimating equation approach. Biometrics. 1988:44:1049-60.

22. Hanley JA, Negassa A, MDd E, Forrester JE. Statistical analysis of correlated data using generalized estimating equations: an orientation. Am J Epidemiol. 2003;157(4):364-75.

23. Hao HX, Xie Y, Zhang Y, Charlat O, Oster E, Avello M, et al. ZNRF3 promotes Wnt receptor turnover in an R-spondin-sensitive manner. Nature. 2012;485(7397):195-200

24. Fuhrmann S. Wnt signaling in eye organogenesis. Organ. 2008;4(2):60-7.

25. Li Q, Weng J, Mohan RR, Bennett GL, Schwall R, Wang ZF, et al. Hepatocyte growth factor and hepatocyte growth factor receptor in the lacrimal gland, tears, and Cornea. Invest Ophthalmol Vis Sci. 1996;37(5):727-39.

26. He PM, He S, Garner JA, Ryan SJ, Hinton DR. Retinal pigment epithelial cells secrete and respond to hepatocyte growth factor. Biochem Biophys Res Commun. 1998:249(1):253-7.

27. Wordinger RJ, Clark AF, Agarwal R, Lambert W, McNatt L, Wilson SE, et al. Cultured human trabecular meshwork cells express functional growth factor receptors. Invest Ophthalmol Vis Sci. 1998;39(9):1575-89.

28. Katoh M. Molecular cloning and characterization of MFRP, a novel gene encoding a membrane-type frizzled-related protein. Biochem Biophys Res Commun. 2001;282(1):116-23.

29. Wang IJ, Lin S, Chiang TH, Chen ZT, Lin LL, Hung PT, et al. The association of membrane frizzled-related protein (MFRP) gene with acute angle-closure glaucoma - a pilot study. Mol Vis. 2008;14:1673-9. 